\title{
48 Übergewicht und Sterberisiko
}

(c) Springer-Verlag GmbH Deutschland, ein Teil von Springer Nature 2018

D. Mathias, Fit und gesund von 1 bis Hundert

https://doi.org/10.1007/978-3-662-56307-6_48

Nach Daten vieler großer Studien ist deutliches Übergewicht mit einem erhöhten Sterberisiko verknüpft (Greenberg 2013; Twig et al. 2016). In einer Metaanalyse aus prospektiven Studien zu dieser Problematik wurden Alter, Geschlecht und Raucherstatus von 894.576 Teilnehmern gesondert berücksichtigt (Whitlock et al. 2009). Die Ergebnisse zeigten dann, dass Fettleibigkeit mit einem BodyMass-Index zwischen 30 und 35 die Lebenserwartung um 2-4 Jahre reduziert. Und es sind gar 8-10 verlorene Lebensjahre, wenn das Gewicht der Menschen zu einem BMI zwischen 40 und 45 führt. Letztere Einschränkung der Lebenserwartung entspricht etwa dem Effekt des Rauchens. Weitere große Metaanalysen liefern ähnliche Ergebnisse, so die Auswertungen von 19 prospektiven Studien mit Daten von fast 1,5 Millionen Erwachsenen europäischer Herkunft und Beobachtungszeiten von 5-28 Jahren durch das US-National Cancer Institute (Gonzales et al. 2010) und die Messwerte von mehr als 10 Millionen Menschen aus vier Kontinenten (The Global BMI Mortality Collaboration, 2016).

Weil der BMI für die Bestimmung der krankmachenden Bauchfettmenge kein optimales Maß ist, können z. B. Übergewichtige mit einer über den ganzen Körper verteilten Fettmasse oder sehr muskuläre Sportler trotz eines erhöhten BMI metabolisch gesund sein und eine längere Lebenserwartung als Normalgewichtige haben (Flegal et al. 2013). Umgekehrt sind bei einer zu großen Ansammlung der Fettpolster in den Bauchorganen, speziell in der Leber, die Sterberaten sogar von Personen mit Normaloder Untergewicht erhöht (Zhang et al. 2008; Cerhan et al. 2014; Tomiyama et al. 2016). Dass es jeweils die zu großen Mengen an Bauchfett sind, die höhere Mortalitätsraten bedingen, wird durch Ergebnisse der Framingham Heart Study (Britton et al. 2013) und der EPIC-Studie bestätigt (van der A et al. 2014).

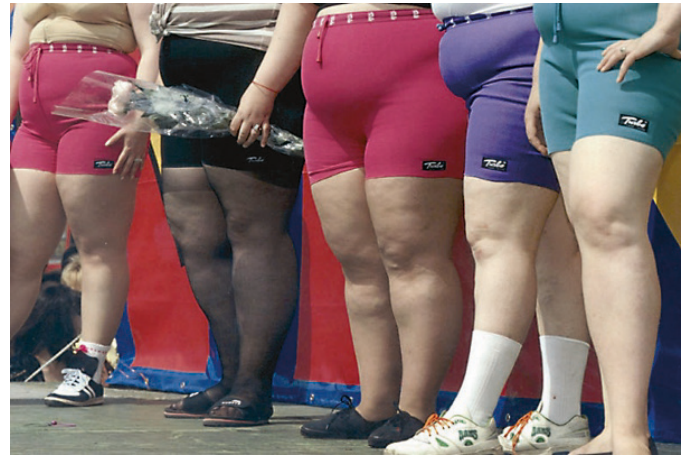

- Abb. 48.1 @ dpa/akg

Ein zu hoher Bauchfettanteil lässt sich durch chirurgische Maßnahmen minimieren. Bei solchen Operationen wird ein Magenbypass angelegt, bei dem nur ein kleiner Teil des Magens für die Nahrungsaufnahme belassen wird oder es wird der Darm verkürzt. Mit diesen Operationen kann dann ein deutlicher Gesundheitsgewinn erzielt werden (Adams et al. 2012; O'Brien et al. 2013; Inge et al. 2016; Schauer et al. 2017). Das bestätigt auch die seit 1981 laufende Swedish Obese Subjects Study über den Zusammenhang zwischen Körpergewicht und Sterblichkeit (Sjöström et al. 2007, 2012, 2014; Carlsson et al. 2012). Die Autoren nutzen hier ebenfalls die Maßnahmen der bariatrischen Chirurgie, um bei Menschen mit krankhafter Adipositas das Gewicht zu senken. Dieses Kollektiv vergleichen sie jeweils mit einem nicht operierten Kollektiv adipöser Personen. Lediglich die operativ versorgten Patienten weisen einen erheblichen Gewichtsverlust auf und nur bei ihnen ist die Inzidenz für Diabetes, Herzinfarkt und Schlaganfall deutlich reduziert sowie die Sterblichkeitsrate klar vermindert. Auch verbessern sich durch bariatrische Operationen langfristig die Gelenkfunktionen (King et al. 2016). 